\title{
Gigantic vulvar masses: a case series of benign large vulvar tumors
}

\author{
Lorie Ann H. Bringas*, Jimmy A. Billod
}

Department of Obstetrics and Gynecology, Baguio General Hospital and Medical Center, Baguio City, Philippines

Received: 11 April 2021

Accepted: 10 May 2021

\section{*Correspondence:}

Dr. Lorie Ann H. Bringas,

E-mail: lahbringasmd@gmail.com

Copyright: ( ) the author(s), publisher and licensee Medip Academy. This is an open-access article distributed under the terms of the Creative Commons Attribution Non-Commercial License, which permits unrestricted non-commercial use, distribution, and reproduction in any medium, provided the original work is properly cited.

\begin{abstract}
Benign vulvar masses are uncommon condition of the lower genital tract. To date, there is no recognized classification for benign tumors. Most of the vulvar tumors show no symptoms, unless large enough to be noted on self-examination. Presented are 3 cases of large vulvar masses. The first case is a Bartholin's gland cyst in 56 years old, G5P4 (4014) with a 5-year history of gradually enlarging mass characterized as fleshy, approximately $16 \times 14 \times 12$ $\mathrm{cm}$, soft, non-tender located in the medial portion of the left labia majora. Grossly, the specimen consists of a unilocular cyst filled with serous fluid and had a smooth glistening inner capsule wall. The second case is a cellular angiofibroma in 61 years old, G0, who developed a fleshy mass, approximately $13 \times 18 \times 15 \mathrm{~cm}$, soft, non-tender, attached to the left labia majora via a pedicle, which developed for 10 years. The specimen is described as an irregular mass with well circumscribed nodules with soft to rubbery, flesh cut surface. The third case is lipoblastoma-like tumor of the vulva in 31 years old, G2P2 (2002), who had a fleshy hypopigmented mass, approximately $15 \times 12 \times 10$ $\mathrm{cm}$, pendulous, soft, smooth, non-tender in the right inferolateral labia majora, which grew for 8 years. The specimen is characterized as an irregular mass partially overlain by skin with cut sections showing cream fatty lobulated surfaces. Benign vulvar masses may present similarly to one another hence careful and detailed assessment should be done. Histologic evaluation is critical in establishing an accurate diagnosis due to the fact that multiple diagnoses may have similar gross characteristics. Management is based on type and size of mass and symptomatology of patient.
\end{abstract}

Keywords: Benign vulvar tumors, Bartholin's gland cyst, Cellular angiofibroma, Lipoblastoma

\section{INTRODUCTION}

Benign tumors of the vulva are relatively infrequent and may show nonspecific clinical manifestations. These tumors are uncommon in the lower genital tract, but are commonly a reason for a gynecologic consultation. ${ }^{1}$ Although most tumors do not require treatment, it is necessary to consider a differential diagnosis that includes infectious lesions, skin cancers, and pre- and malignant tumors. ${ }^{2}$ A biopsy is often needed to exclude a malignant neoplasm to indicate proper treatment.

Vulvar tumors can be basically divided into two major groups: cystic and solid tumors. Within the cystic group is the Bartholin cyst or abscess, epidermoid inclusion cyst, canal of Nuck cyst, Skene (paraurethral) cyst, and vestibular mucous cyst. Solid tumors of the vulva can be divided into three major groups: squamous, glandular, and mesenchymal. ${ }^{2}$

We report three cases of benign vulvar masses with different histopathologic diagnoses, all presented with gradually enlarging mass. The objectives of this case series reports are to: (1) discuss benign vulvar masses and its diagnosis, (2) to discuss individually the different histopathology, and (3) discuss the management.

\section{CASE SERIES}

\section{Case 1}

A 56-year-old, G5P4 (4014) presented with an enlarging left labial mass. The present condition started five years 
prior to admission when she noted a mass on her left labia majora, approximately $3 \times 3 \mathrm{~cm}$ in size, tender. Consult was done at a local health center for which she was given an unrecalled antibiotic. The mass gradually increased but the pain subsided hence the patient was lost to follow up. Interim revealed continuous growth of the vulvar mass with associated occasional dysuria. Patient underwent total hysterectomy with bilateral salpingooophorectomy for ovarian cancer and is currently undergoing chemotherapy.

Review of systems revealed no weight loss, anorexia and body weakness. Systemic physical examination was essentially normal. Examination of the genitalia revealed a fleshy mass, approximately $16 \times 14 \times 12 \mathrm{~cm}$, soft, nontender located in the medial portion of the left labia majora (Figure $1 \mathrm{~A}$ and $\mathrm{B}$ ). Internal examination was unremarkable. The working diagnosis was a vulvar mass left labia majora rule out malignancy. Ancillary procedures were normal. She underwent excision of the vulvar mass under spinal anesthesia. The right labial mass was fleshy, soft measuring $16 \times 14 \times 12 \mathrm{~cm}$, containing serous non-foul-smelling fluid.

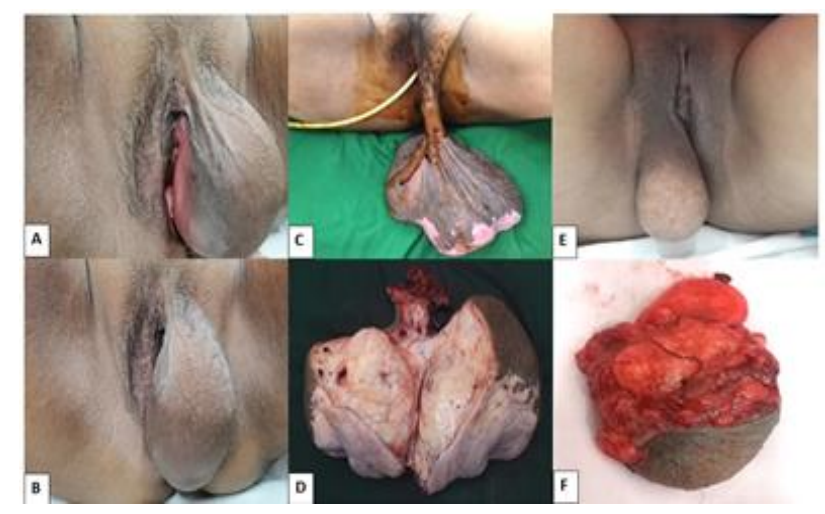

Figure 1: (A, B, C, D, E and F) Gross appearances of the cases. A and B: Bartholin's cyst. Fleshy mass, approximately $16 \times 14 \times 12 \mathrm{~cm}$, soft, non-tender located in the medial portion of the left labia majora. $\mathrm{C}$ and D: Cellular angiofibroma. Fleshy hyperpigmented mass, approximately $13 \times 18 \times 15 \mathrm{~cm}$, soft, non-tender, attached to the left labia majora via a pedicle. $E$ and F: Lipoblastoma like tumor of the vulva. Fleshy mass approximately $15 \times 12 \times 10 \mathrm{~cm}$, soft, pendulous, nontender, with a base attached to the inferolateral portion of the right labia majora.

Histopathologic findings revealed Bartholin cyst. Microscopically, the mass was described to be lined with squamous and urothelial epithelium, but may be destroyed by inflammatory infiltrate (Figure 2).

\section{Case 2}

A 61-year-old, nulligravid presented with a mass in her left labia. The present condition started ten years prior to admission when she noted a mass on her left labia majora, approximately $1 \mathrm{x} 1 \mathrm{~cm}$ in size, slightly tender.
The mass was noted to have enlarged over the years until it reached its present size. There were no consultations done or medications taken for the condition. She is a known hypertensive for 20 years and is maintained on Amlodipine $10 \mathrm{mg} / \mathrm{tab}$ once daily. Her family, social and sexual, gynecologic and obstetric history were unremarkable.

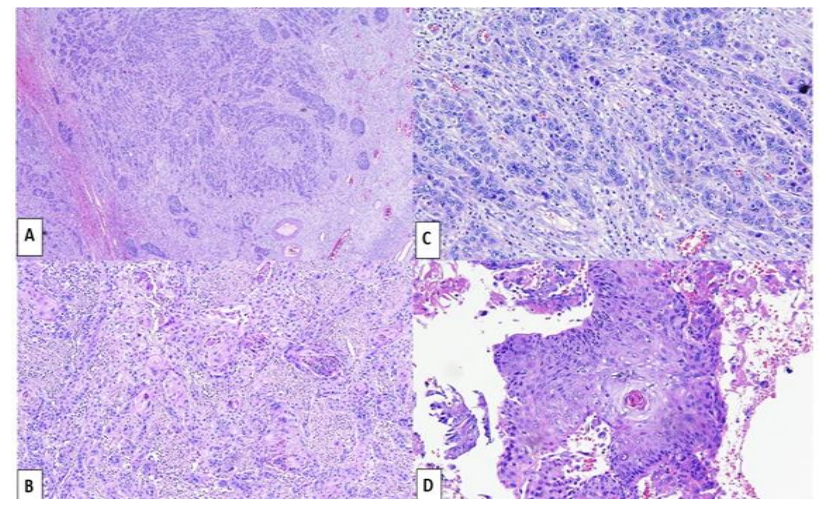

Figure 2: (A, B, C and D), $\mathbf{H}$ and $\mathbf{E}$ staining, case 1 (Bartholinc cyst). Dilated duct lined by transitional epithelium and numerous mucus glands in the cyst wall of the tissues with inflammatory infiltrates.

Review of systems revealed no weight loss, anorexia and body weakness. Systemic physical examination was essentially normal. Examination of the genitalia revealed a fleshy mass, approximately $13 \times 18 \times 15 \mathrm{~cm}$, soft, nontender, attached to the inferior portion of the left labia majora via a pedicle (Figure 1C). Internal examination was unremarkable. The working diagnosis was a Fibroepithelial polyp, left labia majora, rule out malignancy. Laboratory and imaging work ups were normal.

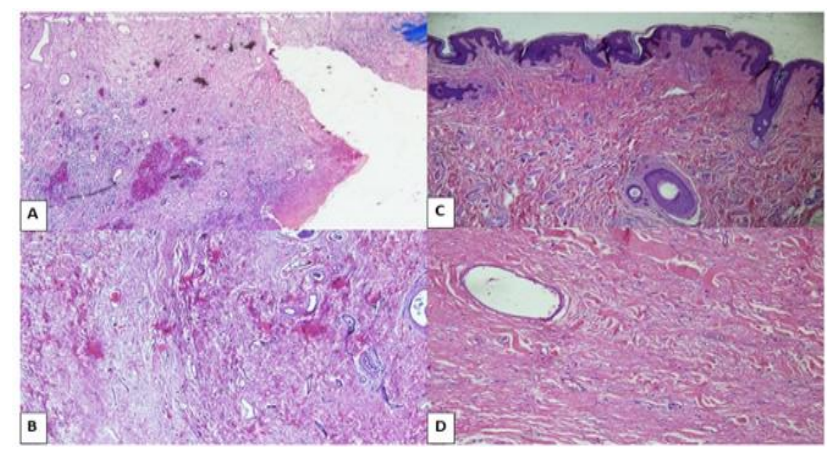

Figure 3: (A, B, C and D), H and E staining, case 2 (Cellular angiofibroma). Bland spindle cells and prominent small to medium-sized vessels with mural hyalinization. Spindle cells are arranged in short intersecting fascicles lying between short bundles of wispy collagen.

She underwent partial simple vulvectomy under spinal anesthesia. The left labial mass was brownish, pedunculated, soft measuring $13 \times 18 \times 15 \mathrm{~cm}$, with a pedicle measuring $7 \times 3 \times 3 \mathrm{~cm}$. On cut section, the mass 
was composed of whitish, glistening, soft tissue (Figure 1D). Histopathologic findings revealed a cellular angiofibroma. Microscopically, bland spindle cells and prominent small to medium-sized vessels with mural hyalinization can be seen. The spindle cells are arranged in short intersecting fascicles lying between short bundles of wispy collagen (Figure 3).

\section{Case 3}

A 31-year-old, G2P2 (2002), presented with an 8-year history of gradually enlarging non-tender mass in her right labia initially approximately $3 \times 3 \mathrm{~cm}$ in size. The mass gradually enlarged reaching its present size. There were no previous consultations done or medications taken for the condition. Past medical, family, social and sexual, gynecologic and obstetric history were non-contributory.

Review of systems revealed no weight loss, anorexia and body weakness. Systemic physical examination was essentially normal. Examination of the genitalia revealed a fleshy skin tone mass, approximately $15 \times 12 \times 10 \mathrm{~cm}$, pendulous, soft, smooth, non-tender in the right inferolateral labia majora (Figure 1E). Internal examination was unremarkable. The working diagnosis was a Vulvar mass right labia majora to consider lipoma, rule out malignancy. Ancillary tests were normal. She underwent excision of the mass under spinal anesthesia. The right labial mass was fleshy, soft measuring
$18 \times 15 \times 15 \mathrm{~cm}$, on cut section, the mass was composed of yellow, adipose and soft tissue (Figure 1F). Histopathologic findings revealed a lipoblastoma like tumor. Microscopically, the mass was described to have both mature adipocytes and lipoblasts with myxoid stroma and plexiform vascular network (Figure 4). Table 1 presents the summary of the three cases presented.

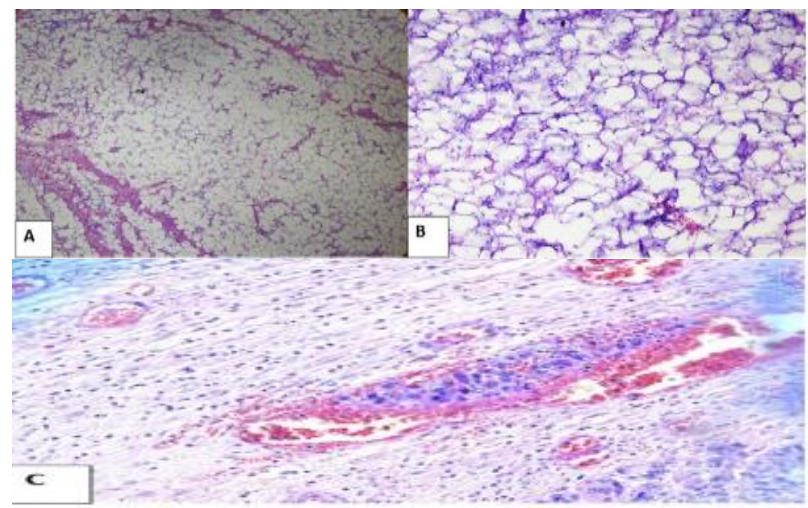

Figure 4: (A, B and C), $\mathrm{H}$ and $\mathrm{E}$ staining, case 3 (Lipoblastoma like tumor of the vulva). Lobulated and composed of variable proportions of mature adipocytes, bland uni-vacuolated and bi-vacuolated lipoblasts, and spindle cells with short stubby nuclei. These cells to be diffusely scattered in a myxoid background with prominent branching vessels.

Table 1: Summary of cases, clinical and pathologic features.

\begin{tabular}{|c|c|c|c|}
\hline Characteristics & $\begin{array}{l}\text { Case } 1 \\
\text { (Bartholin's cyst) }\end{array}$ & $\begin{array}{l}\text { Case } 2 \\
\text { (Cellular angiofibroma) }\end{array}$ & $\begin{array}{l}\text { Case } 3 \\
\text { (Lipoblastoma like tumor) }\end{array}$ \\
\hline Age (years) & 56 & 61 & 31 \\
\hline $\begin{array}{l}\text { Time elapsed for tumor } \\
\text { growth (years) }\end{array}$ & 5 & 10 & 8 \\
\hline Symptoms & $\begin{array}{l}\text { Vulvar mass } \\
\text { Occasional dysuria }\end{array}$ & Vulvar mass & Vulvar mass \\
\hline Procedure & Excision of vulvar mass & Partial simple vulvectomy & Excision of vulvar mass \\
\hline Gross appearance & $\begin{array}{l}\text { Fleshy mass, approx. } \\
16 \times 14 \times 12 \mathrm{~cm} \text {, soft, non- } \\
\text { tender located in medial } \\
\text { portion of left labia majora }\end{array}$ & $\begin{array}{l}\text { Fleshy mass, approximately } \\
13 \times 18 \times 15 \mathrm{~cm} \text {, soft, non-tender, } \\
\text { attached to the left labia majora } \\
\text { via a pedicle }\end{array}$ & $\begin{array}{l}\text { Fleshy skin tone mass, } \\
\text { approx. } 15 \times 12 \times 10 \mathrm{~cm} \text {, } \\
\text { pendulous, soft, smooth, } \\
\text { non-tender in right } \\
\text { inferolateral labia majora }\end{array}$ \\
\hline $\begin{array}{l}\text { Microscopic } \\
\text { appearance }\end{array}$ & $\begin{array}{l}\text { Lined with squamous and } \\
\text { urothelial epithelium with } \\
\text { inflammatory infiltrates } \\
\text { which may have } \\
\text { calcifications resembling } \\
\text { malakoplakia }\end{array}$ & $\begin{array}{l}\text { Bland spindle cells and prominent } \\
\text { small to medium-sized vessels } \\
\text { with mural hyalinization. Spindle } \\
\text { cells are arranged in short } \\
\text { intersecting fascicles lying } \\
\text { between short bundles of wispy } \\
\text { collagen }\end{array}$ & $\begin{array}{l}\text { Contains both mature } \\
\text { adipocytes and lipoblasts } \\
\text { with myxoid stroma and } \\
\text { plexiform vascular network }\end{array}$ \\
\hline
\end{tabular}

\section{DISCUSSION}

Benign vulvar masses are common in adult women and frequently a reason for a gynecologic consultation. However, the frequency of tumors (benign as well as malignant) is very low. Their incidence has not been reported much. This field despite being uncommon has not been explored. ${ }^{3}$ Although most tumors do not require treatment, it is necessary to consider a differential diagnosis that includes infectious lesions, skin cancers, and pre- and malignant tumors. Patients usually undergo excision due to cosmesis, clothing friction and associated 
symptomatology. Currently, there is no established classification of benign tumors of the vulva. Clinically, however, vulvar tumors can be simply divided into two major groups: cystic and solid tumors.1

The vulvar zone contains dense apocrine glands, anogenital mammary like glands along with eccrine glands and folliculosebaceous units. Generally cystic tumors are described as a mass containing a sac that may be filled with air, fluid or other material, in contrast to solid tumors any abnormal mass of tissue or swelling with no other component. ${ }^{4}$

\section{Bartholin's cyst}

Cysts and abscess of the Bartholin's gland are the most common disorders of the vulva and are usually present in a woman's reproductive period. When the ducts become obstructed, mucous or pus accumulates leading to dilation and enlargement of the gland. A Bartholin cyst is associated with a viscous syrupy fluid that fills the gland. They are typically 1 to $3 \mathrm{~cm}$ in size but can measure up to $5 \mathrm{~cm}$. Small cysts may not cause symptoms. Larger cysts may cause pressure and pain. ${ }^{5}$

The most common types of Bartholin's gland masses are cysts or abscesses, with abscesses occurring three times more frequently than cysts, Bartholin's duct cysts typically range from 1 to $4 \mathrm{~cm}$ in diameter, and much larger ones are rare. ${ }^{5}$ The most commonly described etiological factor is perineal trauma. Our patient had no history of genital trauma. The majority are unilateral, tender cystic masses located in the lower medial labia majora, as in this case. Patients can have diverse symptoms, ranging from mild discomfort to urinary symptoms such as obstructed voiding which was present in our patient.

The differential diagnosis of a Bartholin's cystic mass should be considered with other vulvar pathologies, such as epidermoid or epidermal inclusion cyst, mucinous or ciliated cysts, canal of Nuck cyst, Skene's paraurethral gland cyst, vaginal wall cysts, lipoma, endometrioma, dermoid cyst, post-traumatic hematoma, inguinal hernia and vulvar malignant tumor. The key to identifying a Bartholin's duct cyst is the anatomical location of the mass, with a final histopathological examination required for differentiation from other vulvar lesions. ${ }^{5}$

Malignant counterpart of the Bartholin gland cyst is the Bartholin gland carcinomas (BGCs) which are extremely rare tumors, accounting for $<1 \%$ of all female genital malignancies. BGCs commonly occur in postmenopausal female with a median age of 50 years at presentation. The pathogenesis of BGC remains unknown; however, it may be associated with infections of the vulva and Bartholin glands. 6

Imaging studies by ultrasound, computed tomography or magnetic resonance may be required to characterize the lesion further. Though magnetic resonance is useful in guiding treatment planning, especially for the detection of extension into deep perineal tissue if the cyst or abscess is large, surgical excision and pathological examination are necessary for diagnosis. ${ }^{5}$ In our case, no other imaging tests were performed because the physical exploration did not raise concerns.

Microscopic examination of a typical Bartholin's gland reveals acini lined by columnar, mucus-secreting epithelium and ducts lined by transitional epithelium. Larger ducts can contain areas of stratified squamous epithelium. Thus, cysts arising from the main duct are lined by transitional or squamous epithelium, whereas cysts originating in an acinus are lined by mucinous columnar epithelium. ${ }^{6}$ In the case of our patient, it is lined by transitional epithelium.

Therapeutic considerations are not usually complex. There are many treatment options, including antibiotics, simple drainage, fistulization, marsupialization or excision of the gland. The preferred treatment is surgical drainage and marsupialization, as it preserves function and prevents reformation of the cyst or abscess. Recurrence is reported in approximately $20 \%$ of patients (ranging from $0 \%$ to $38 \%$ ), and is more frequent after simple drainage. ${ }^{6}$ Fistulization and marsupialization give a very low rate of recurrence, although the rate is probably underestimated due to a short follow-up time. ${ }^{5,6}$

Antibiotics are frequently included in the treatment as a second-line therapy or in addition to surgical procedures. Less than $20 \%$ of cultures from Bartholin's cysts and approximately $67 \%$ of cultures from Bartholin's abscesses indicate infection. The etiology of Bartholin's gland abscesses is known to be polymicrobial, mainly with opportunistic bacteria. Recent studies indicate that Escherichia coli was the single most frequent pathogen in cultures of acute Bartholin's abscess. Thus, amoxicillinclavulanate could be an appropriate choice of antimicrobial agent. In the reported case, antibiotics were not maintained after the intervention because the patient was lost to follow up.

\section{Cellular angiofibroma}

Cellular angiofibroma (CA) belongs to soft tissue tumors that predominantly occur in the distal genital tract of both genders. The first soft tissue tumor with a relatively pelvic site-specificity was a benign stromal polyp described in the early 1960. Among the mesenchymal tumors involving the vulva, we can distinguish the lesions that can arise at any site, including the vulva, and those more characteristics of this area.

Cellular angiofibroma of the vulva is a benign mesenchymal tumor of the vulva with histologic features suggestive of spindle cell lipoma, and was first described in 1997. ${ }^{7}$ The term cellular angiofibroma was chosen to emphasize the cellular spindle cell component and the 
prominent blood vessels characteristic of this tumor. This type of tumor was characteristically reported in middle-aged women; however, according to recent literature, both women and men seem to be equally affected, most often in the fifth and seventh decades, respectively. In women, this tumor usually arises in the subcutaneous tissues of the vulva and presents as a small $(<3 \mathrm{~cm})$ mass often described as a painless swelling, however in our patient the mass started as a small painless swelling which grew overtime. ${ }^{5}$

Diagnosis of soft tissue tumors of the vulva can be challenging and frequently necessitates the use of CT or MRI for further characterization and to aid surgical planning. Cellular angiofibroma are almost always encapsulated, well circumscribed, and hypervascular; thus, surgical removal of these tumors requires thorough knowledge of pelvic anatomy to minimize blood loss. ${ }^{6,7}$

Cellular angiofibroma in women represents a distinct benign neoplasm with a broad anatomic distribution even if it is mainly localized in the vulvo-vaginal area. This lesion may exhibit some variations in its phenotypic features, as well as atypia and morphologic features of sarcomatous transformation but these characteristics seem not to predispose to a malignant fashion and recurrences. $^{8}$

Histologically, the tumor is composed of a uniform, monotonous spindle cell proliferation, often admixed with prominent vessels with thick and hyalinized walls, and clusters of mature adipocytes. It is usually well circumscribed but can be focally infiltrative, although it is not associated with aggressive clinical behavior.

Local excision with clear margins seems to be adequate in the treatment of these lesions; however, data on longterm follow up is not currently available. Surgical removal of the mass with its capsule not only helps guarantee complete excision and prevent its recurrence but also minimizes blood loss. ${ }^{8}$

\section{Lipoblastoma-like tumor of the vulva}

Another benign solid tumor of the vulva is Lipoblastomalike tumor of the vulva (LLTV). It is an exceptionally rare adipocytic mesenchymal tumor. These tumor types were first described by in 2002, affecting mostly young adult patients, have a benign clinical course, and share certain histopathologic features with lipoblastoma, myxoid liposarcoma, and spindle cell lipoma (SCL). ${ }^{9}$ In a report of eight cases who have lipoblastoma like tumor of the vulva who underwent excision, none of the patients had developed recurrence after a significant follow-up time interval. The authors concluded that LLTV was a distinct neoplasm akin to lipoblastoma on the basis of the striking morphologic similarity and benign clinical behavior. ${ }^{10}$
Lipoblastoma-like tumor of the vulva is an exceptionally rare adipocytic mesenchymal tumor. These tumors, seem to affect mostly young adult patients, have a benign clinical course, and share certain histopathologic features with lipoblastoma, myxoid liposarcoma, and spindle cell lipoma (SCL). ${ }^{10}$

Histologically, LLTVs are lobulated and composed of variable proportions of mature adipocytes, bland univacuolated and bi-vacuolated lipoblasts, and spindle cells with short stubby nuclei in a diffusely myxoid background with prominent branching vessels. Nuclear atypia is minimal. No necrosis and only rare mitotic activity should be identified. ${ }^{9}$ Conservative but complete excision was recommended as optimal treatment as what was done in the case of our patient.

\section{CONCLUSION}

Benign vulvar masses may present similarly to one another hence careful and detailed assessment should be done. Correct diagnosis is an indispensable part in the gynecologic assessment and management. It is essential for the gynecologist to differentiate between benign and malignant lesions to provide an appropriate treatment. Because multiple diagnoses may have similar gross characteristics, histologic evaluation is critical in establishing an accurate diagnosis. Histologic classification based on the tissue of origin is practical in the description of vulvar lesions. Management is based on the type and size of the mass and symptomatology of the patient. Most of the large benign vulvar mass are treated with excision with very low recurrence rate.

\section{Funding: No funding sources \\ Conflict of interest: None declared \\ Ethical approval: Not required}

\section{REFERENCES}

1. Maldonado VA. Benign vulvar tumors. 2014. Available at: https://www.ncbi.nIm.nih.gov/pubmed25220103. Accessed on August 20, 2019.

2. Horbelt DV, Delmore JE. Benign Neoplasms of the Vulva. The Global Library of Women's Medicine. 2009.

3. Sigamani K, Thukkaram C, Harke A. ClinicoPathological Study Of Skin Adnexal Tumours In A Tertiary Care Hospital. J Evolution Med Dental Sci. 2016;5(47):3017-20.

4. Subhawong TK, Fishman EK, Swart JE, Carrino JA, Attar S, Fayad LM. Soft-Tissue Masses and Mass like Conditions: What Does CT Add to Diagnosis and Management? Am J Roentgenol. 2010;194(6):1559-67.

5. Noval BDDL, Fernández IG, Fernández BÁ. Bulky Bartholin's gland cyst: Case report of an incidental finding. Case Rep Women's Health. 2019;22. 
6. Zhan P, Li G, Liu B, Mao X-G. Bartholin gland carcinoma: A case report. Oncol Letters. 2014;8(2):849-51.

7. Khmou M, Lamalmi N, Malihy A, Rouas L, Alhamany Z. Cellular angiofibroma of the vulva: a poorly known entity, a case report and literature review. BMC Clin Pathol. 2016;16:1.

8. Mandato VD, Santagni S, Cavazza A, Aguzzoli L, Abrate M, Sala GBL. Cellular angiofibroma in women: a review of the literature. Diagnostic Pathol. 2015;10:1.
9. Lae ME, Pereira PF, Keeney GL, Nascimento AG. Lipoblastoma-like tumour of the vulva: report of three cases of a distinctive mesenchymal neoplasm of adipocytic differentiation. Histopathology. 2002;40(6);505-9.

10. Mirkovic J, Fletcher CD. Lipoblastoma-like Tumor of the Vulva. Am J Surg Pathol. 2015;39(9):1290-5.

Cite this article as: Bringas LAH, Billod JA.

Gigantic vulvar masses: a case series of benign large vulvar tumors. Int J Reprod Contracept Obstet Gynecol 2021;10:2476-81. 